\title{
Stereoselective Total Synthesis of cis- and trans-3-Hydroxypipecolic Acid
}

\author{
Ningning Liang and Apurba Datta* \\ Department of Medicinal Chemistry, The University of Kansas, \\ 1251 Wescoe Hall Drive, Lawrence, KS 66045, USA \\ adutta@ku.edu
}

\section{Supporting Information}

\section{Table of Contents}

$\begin{array}{ll}\text { General Experimental Details } & \mathrm{S} 1 \\ { }^{1} \mathrm{H} \&{ }^{13} \mathrm{C} \text { NMR spectra of } \mathbf{3} & \mathrm{S} 2 \\ { }^{1} \mathrm{H} \&{ }^{13} \mathrm{C} \text { NMR spectra of } \mathbf{6} & \mathrm{S} 3 \\ { }^{1} \mathrm{H} \&{ }^{13} \mathrm{C} \text { NMR spectra of } \mathbf{7} & \mathrm{S} 4 \\ { }^{1} \mathrm{H} \&{ }^{13} \mathrm{C} \text { NMR spectra of } \mathbf{8} & \mathrm{S} 5 \\ { }^{1} \mathrm{H} \&{ }^{13} \mathrm{C} \text { NMR spectra of } \mathbf{9} & \mathrm{S} 6 \\ { }^{1} \mathrm{H} \&{ }^{13} \mathrm{C} \text { NMR spectra of } \mathbf{1 0} & \mathrm{S} 7 \\ { }^{1} \mathrm{H} \&{ }^{13} \mathrm{C} \text { NMR spectra of } \mathbf{3 A} & \mathrm{S} 8 \\ { }^{1} \mathrm{H} \text { NMR spectra of } \mathbf{3 A}(\mathrm{H}-5, \mathrm{H}-1 \text { ' decoupled) } & \mathrm{S} 9 \\ { }^{1} \mathrm{H} \&{ }^{13} \mathrm{C} \text { NMR spectra of } \mathbf{1 2} & \mathrm{S} 10 \\ { }^{1} \mathrm{H} \&{ }^{13} \mathrm{C} \text { NMR spectra of } \mathbf{1 3} & \mathrm{S} 11 \\ { }^{1} \mathrm{H} \mathrm{\&}{ }^{13} \mathrm{C} \text { NMR spectra of } \mathbf{4} & \mathrm{S} 12 \\ { }^{1} \mathrm{H} \&{ }^{13} \mathrm{C} \text { NMR spectra of } \mathbf{1 4} & \mathrm{S} 13 \\ { }^{1} \mathrm{H} \&{ }^{13} \mathrm{C} \text { NMR spectra of } \mathbf{1 5} & \mathrm{S} 14 \\ { }^{1} \mathrm{H} \mathrm{\&}{ }^{13} \mathrm{C} \text { NMR spectra of } \mathbf{1 6} & \mathrm{S} 15 \\ { }^{1} \mathrm{H} \mathrm{\&}{ }^{13} \mathrm{C} \text { NMR spectra of } \mathbf{1 7} & \mathrm{S} 16 \\ { }^{1} \mathrm{H} \&{ }^{13} \mathrm{C} \text { NMR spectra of } \mathbf{1 8} & \mathrm{S} 17\end{array}$

\section{General experimental details:}

All of the solvents and reagents used were obtained commercially and used as such, unless noted otherwise. Moisture or air sensitive reactions were conducted under argon atmosphere in oven dried $\left(120^{\circ} \mathrm{C}\right)$ glass apparatus. Diethyl ether and THF were distilled from sodium benzophenone ketyl, while dichloromethane was distilled over calcium hydride, prior to use. Solvents were removed under reduced pressure using standard rotary evaporators. Flash column chromatography' was carried out using Silica gel 60 (230-400 mesh), while thin layer chromatography (tlc) was carried out on Silica Gel HLF, pre-coated glass plates. All yields reported refer to isolated material judged to be homogeneous by tlc and NMR spectroscopy. Unless noted otherwise, NMR spectra were recorded with the chemical shifts $(\delta)$ reported in ppm relative to $\mathrm{Me}_{4} \mathrm{Si}$ (for ${ }^{1} \mathrm{H}$ ) and $\mathrm{CDCl}_{3}$ (for ${ }^{13} \mathrm{C}$ ) as internal standards respectively. 


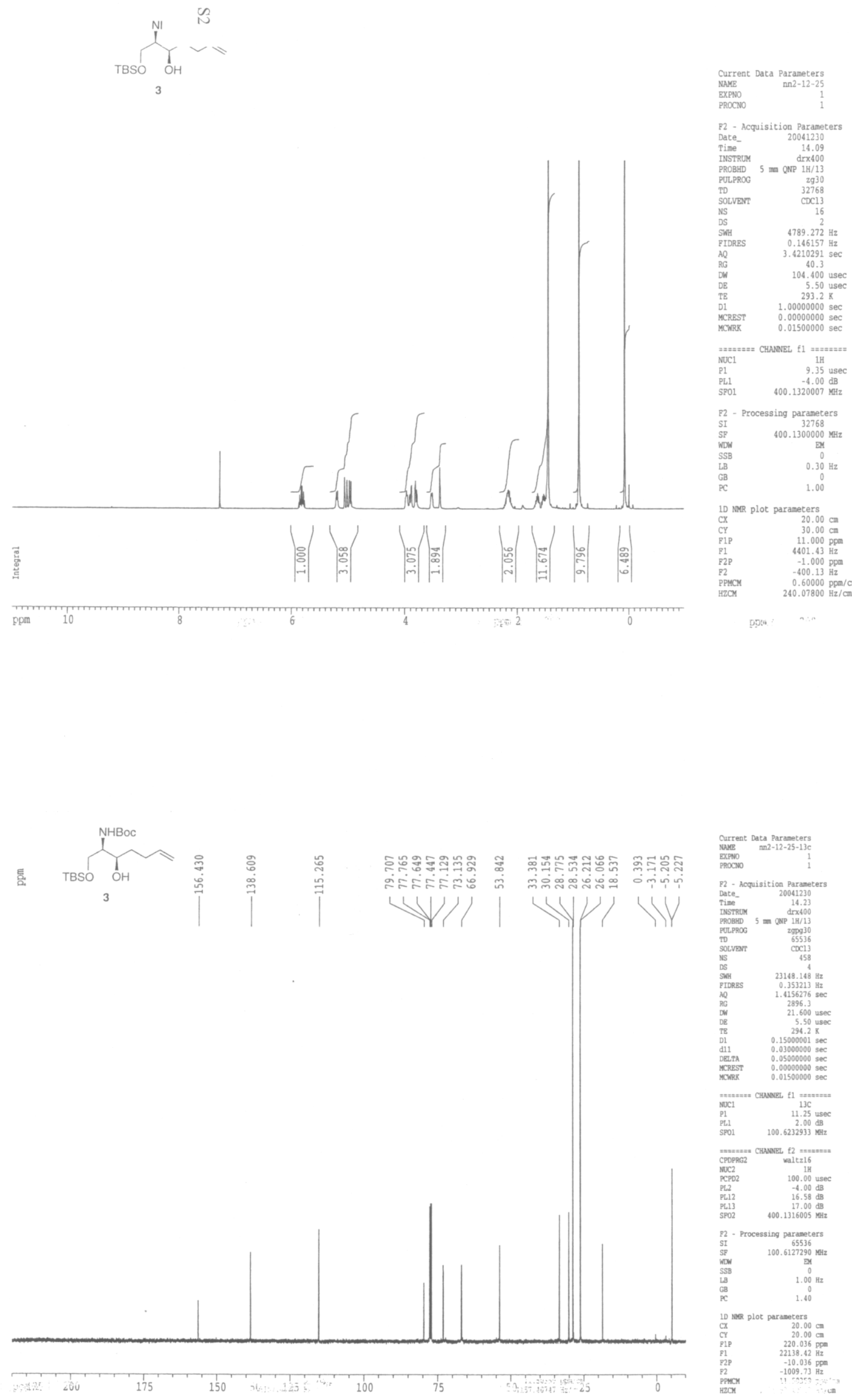



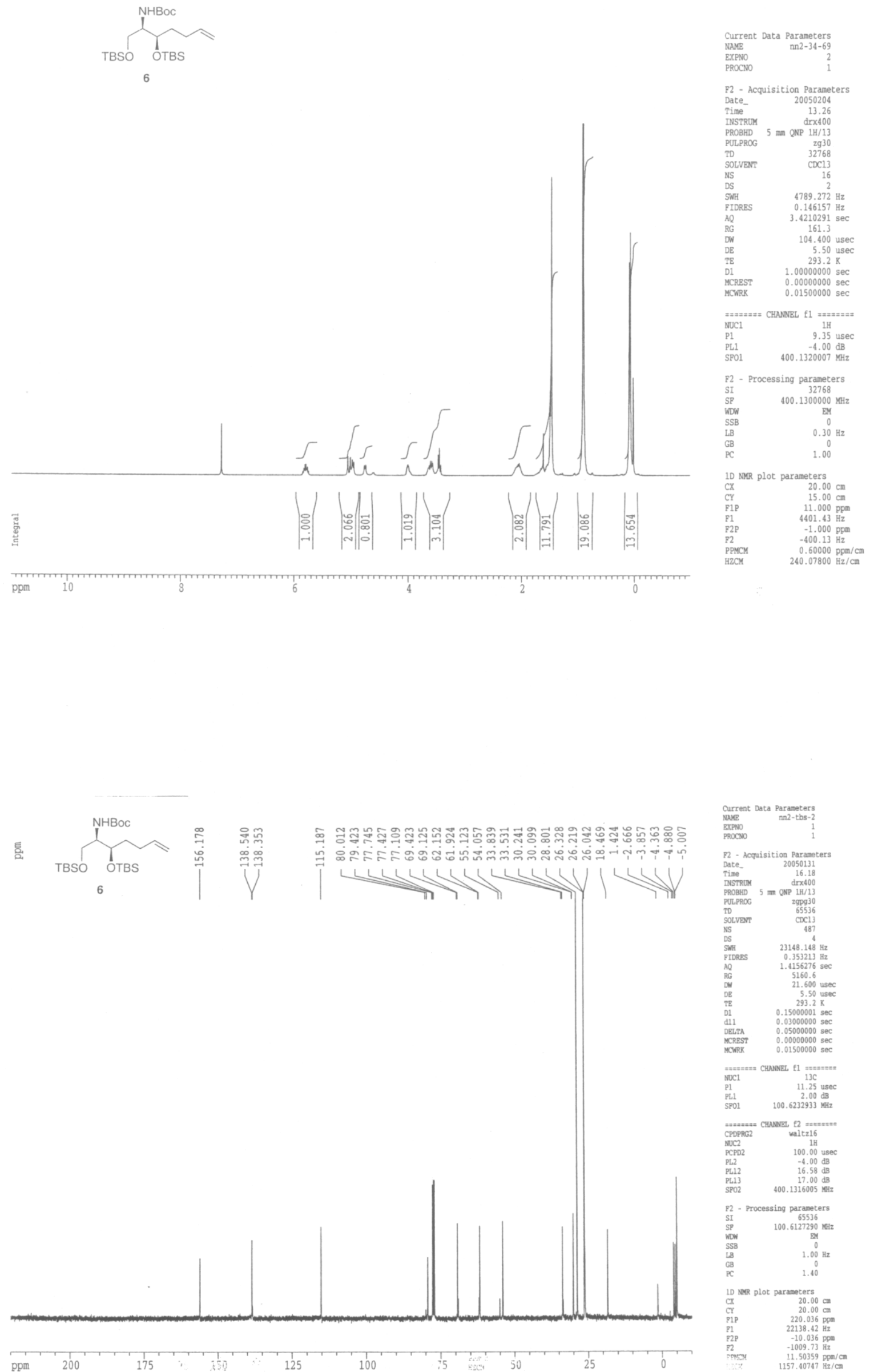

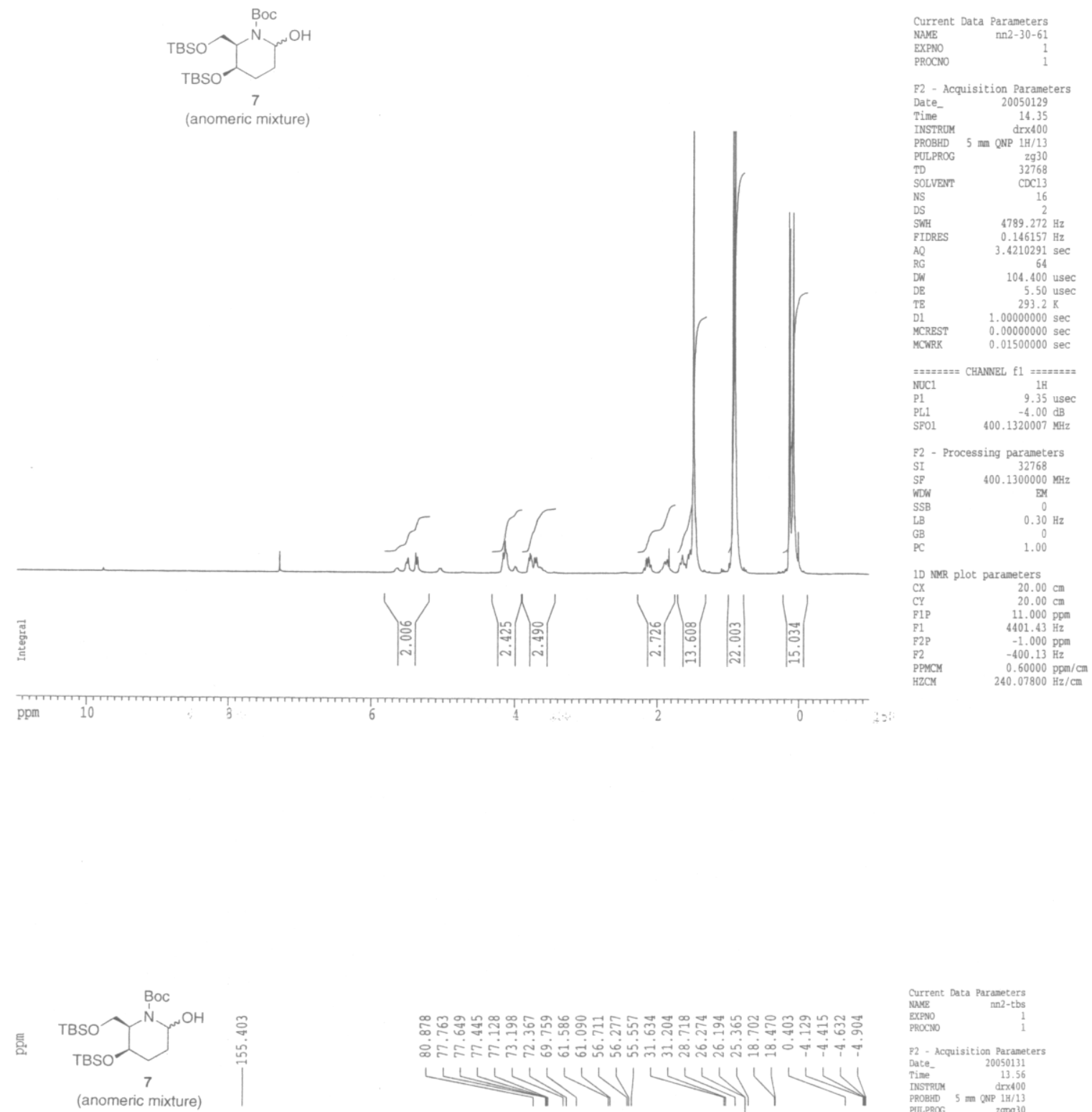

Current Data Parameters

NAME
EXPNO
PROCDO

P2 - Acquisition Parameters

$\begin{array}{lr}\text { Pate - } & 20050131 \\ \text { Dacer } & 13.56 \\ \text { Tine } & \end{array}$

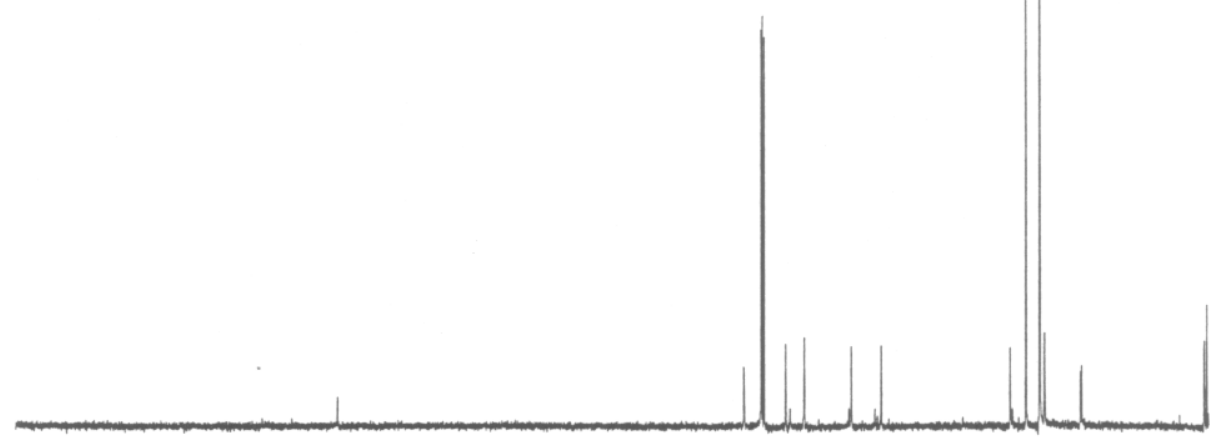

ppies 200

175

150

100

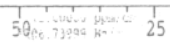

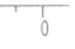

-

$$
\text { (n) }
$$$$
\text { (2) }
$$ 

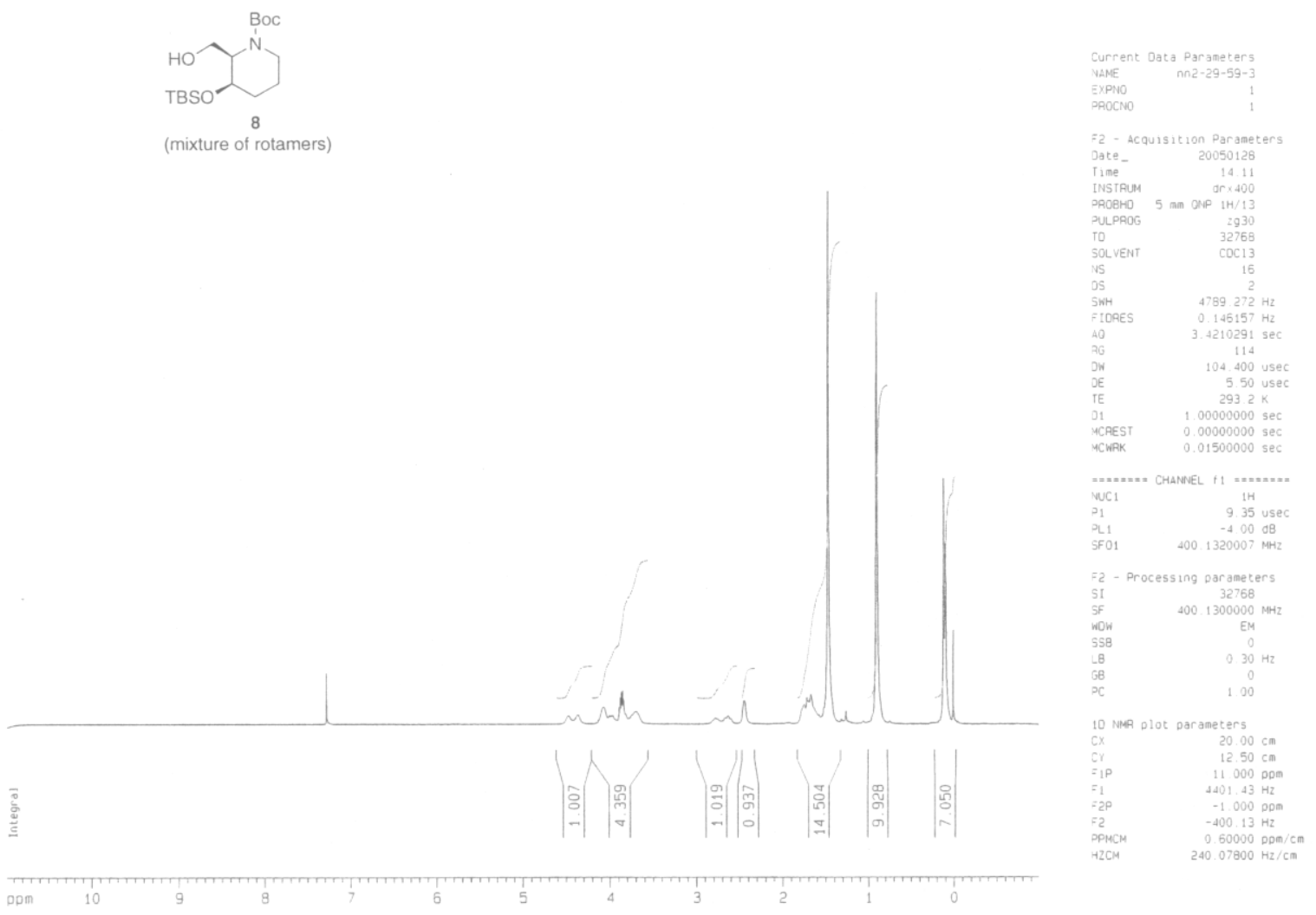

AAC13CPD CDC13 u cstauffe 5
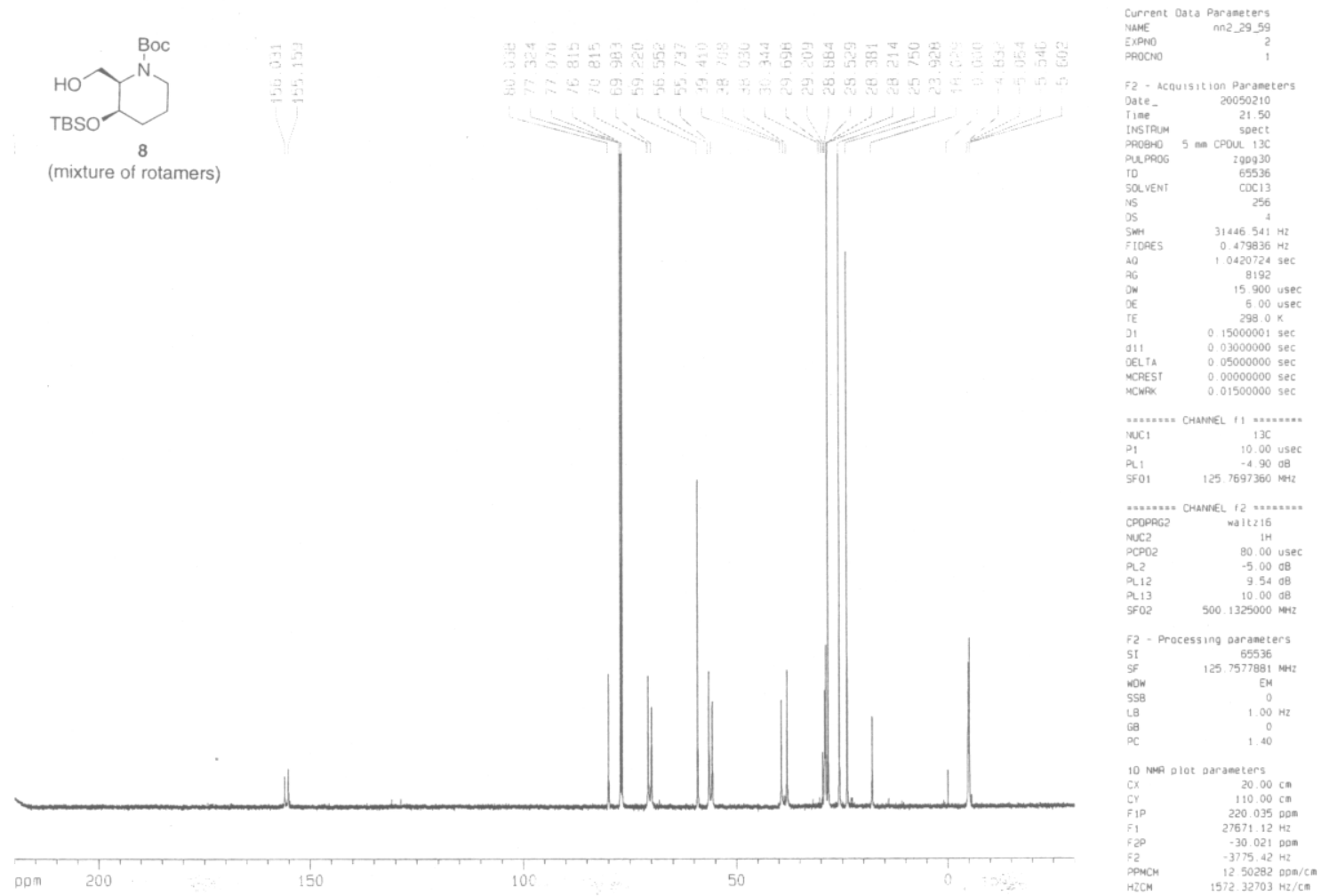

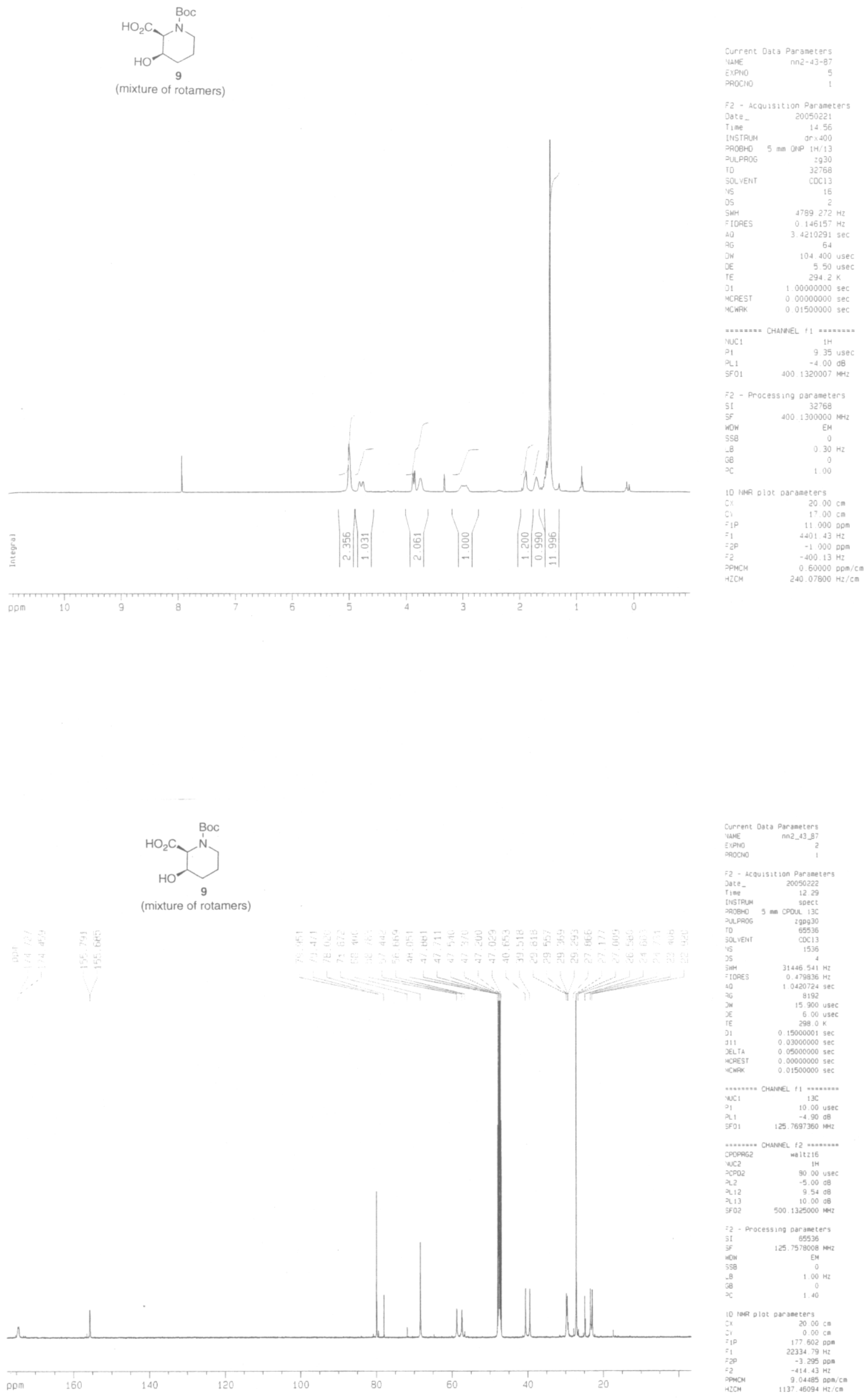

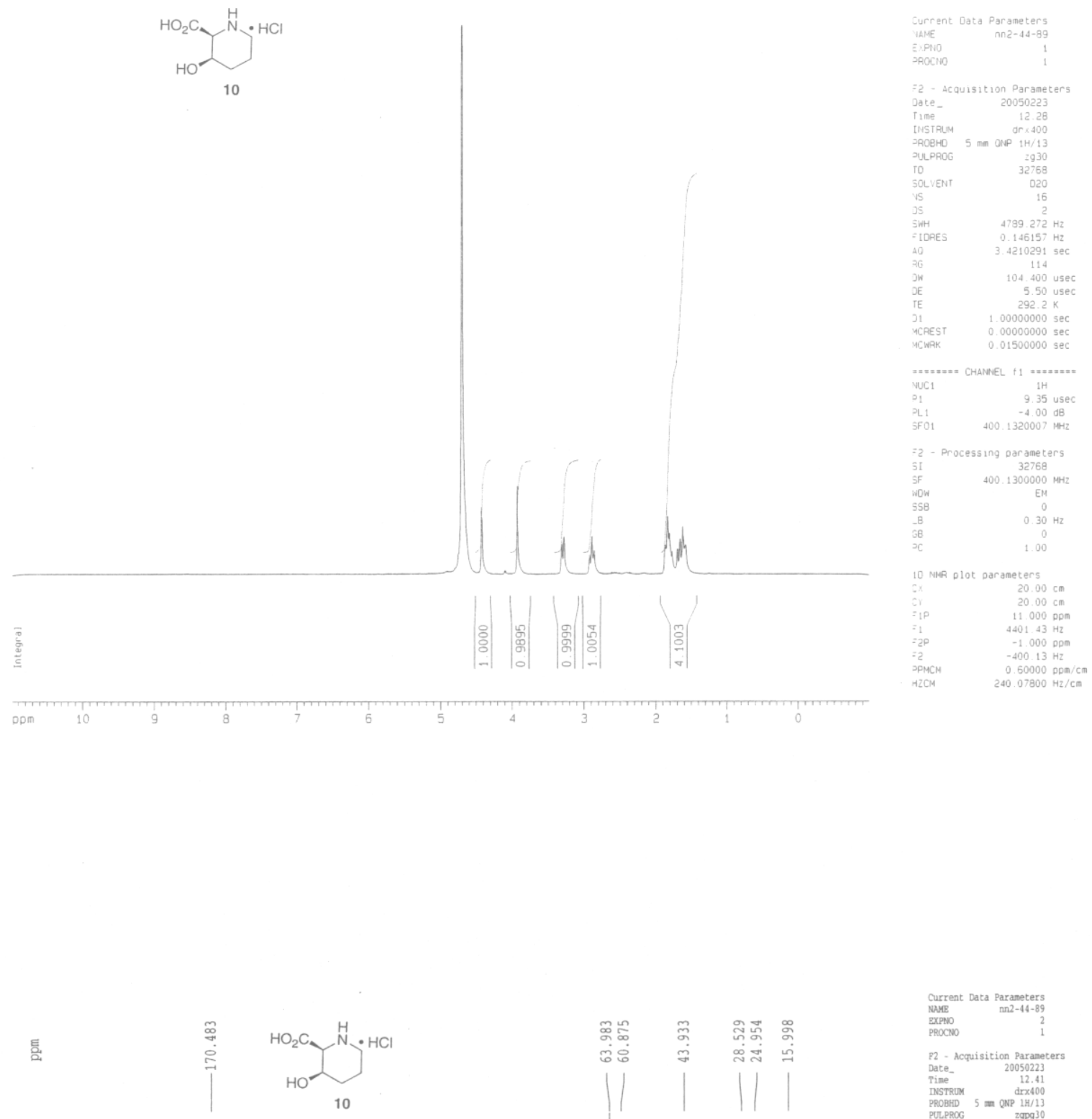

P2 - Acquisition Parameters

$\begin{array}{lr}\text { Date_ } & 20050223 \\ \text { Time } & 12.41 \\ \text { puspres } & \text { d.400 }\end{array}$

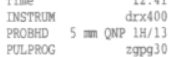

$\begin{array}{lr}\text { 29pg 30 } \\ \text { POLPROC } & 65536 \\ \text { Solverr } & \text { CDC13 } \\ \text { NS } & 451 \\ \text { DS } & 4 \\ \text { SWH } & 23148.148\end{array}$

\begin{tabular}{lr} 
DS & 44 \\
SWH & $23148.148 \mathrm{~Hz}$ \\
PIDRBS & $0.353213 \mathrm{~Hz}$ \\
\hline W & $1.456276 \mathrm{sz}$
\end{tabular}

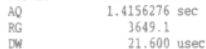

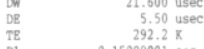

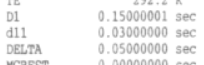

$\begin{array}{ll}\text { MCREST } & 0.00000000 \mathrm{sec} \\ \text { MCWRK } & 0.01500000 \mathrm{sec}\end{array}$

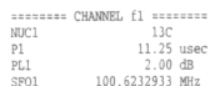

100.6232933 Mis

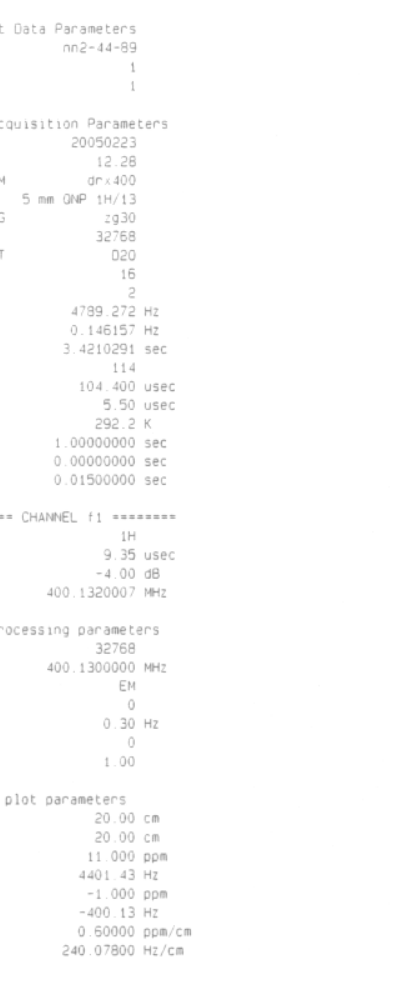

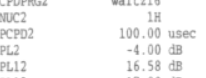

$16.58 \mathrm{~dB}$
$\mathrm{PL13}$
$\mathrm{PL}$

P2.
SI
S9

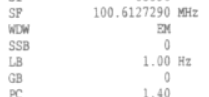

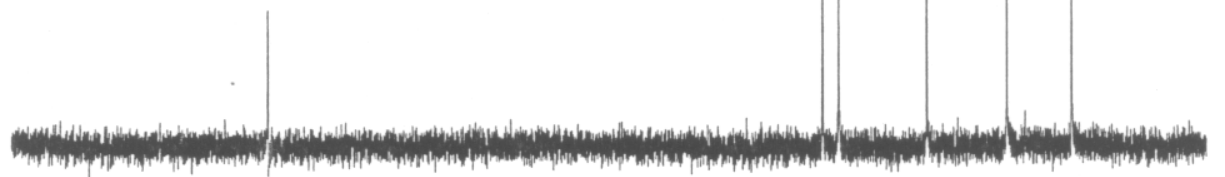

ppm

150

$125 \div 25$

75

50

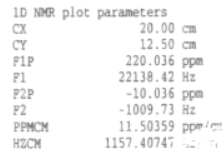



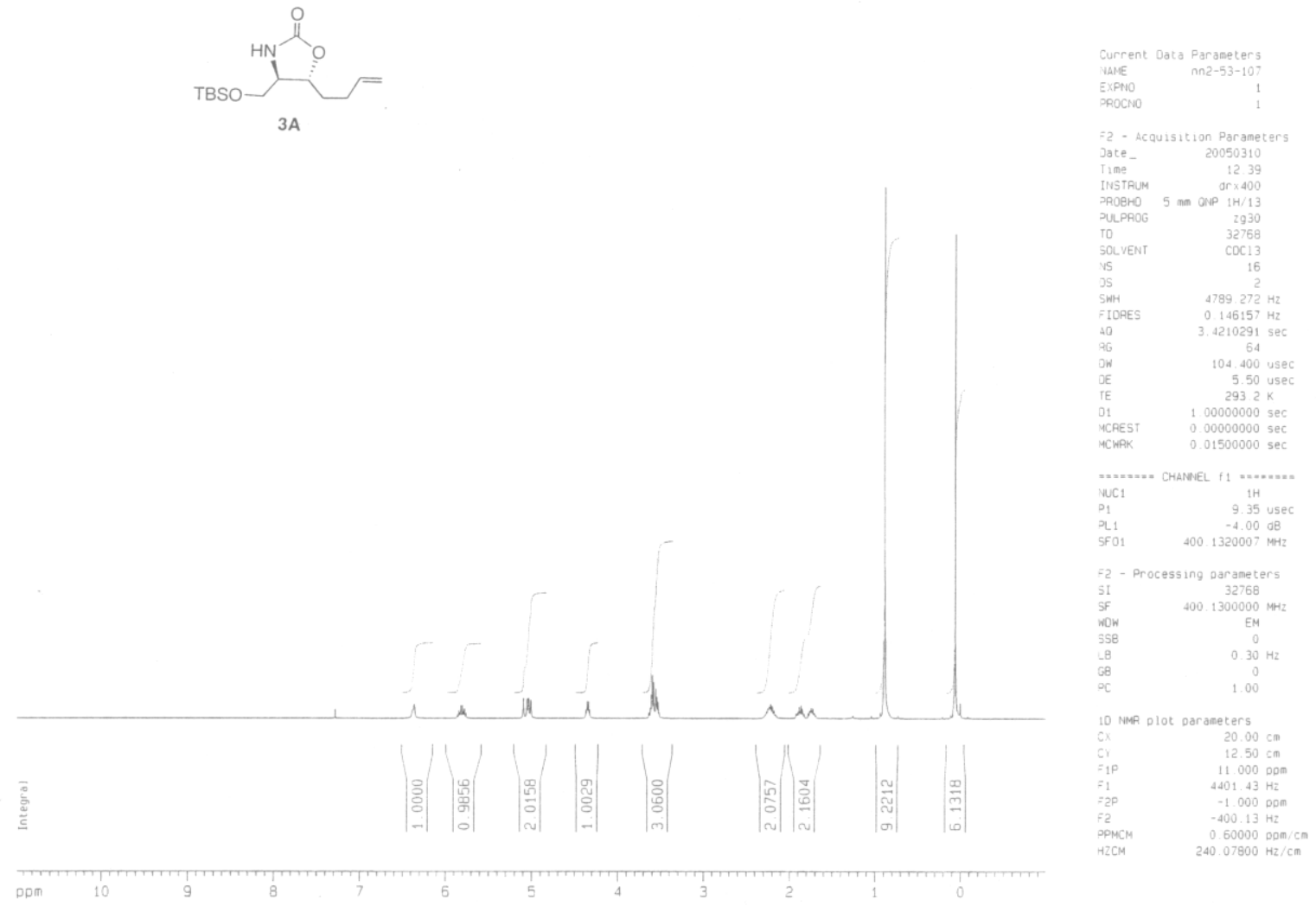

AAC13CPO COC13 u nliang 4

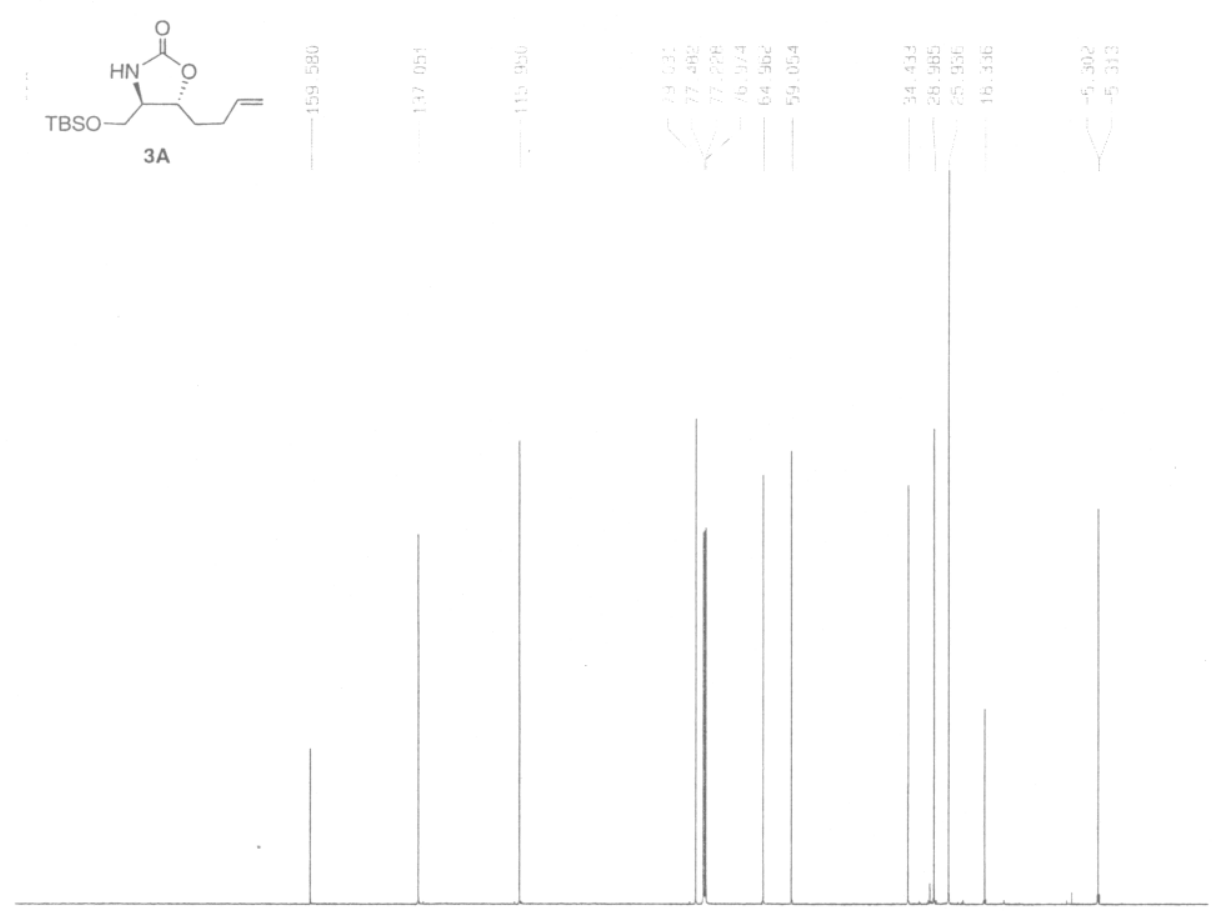

Current Data Parameters

Expro

PPOC

F2 - Acquisition paraseters

20050809
Pate-
Time

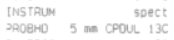

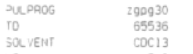

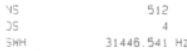

Tores $\quad 0.479936$ he

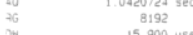

15.900 usec
$x \in$$\quad 600$ usec

$\begin{array}{ll}21 & 0.15000001 \mathrm{sec} \\ 21 & 0.03000000 \mathrm{sec}\end{array}$

Jelit

$\begin{array}{lll} & 0.00000000 \mathrm{sec} \\ \text { Whent } & 0.01500000 \mathrm{sec}\end{array}$

......... OAanel +1 .........

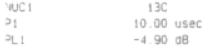

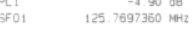

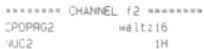

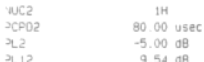

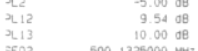

300.1325000 ind

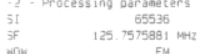

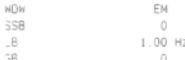

$\begin{array}{lll}36 & 1.40 \\ x & 1.00\end{array}$

10 tade olot parameters
$20,00 \mathrm{col}$

221.626 gon
$=19$
$=1971.18 \mathrm{~Hz}$

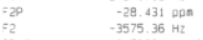

50 $12.50284 \mathrm{pom} / \mathrm{Cm}$
$572.32703 \mathrm{~Hz} / \mathrm{cm}$ 

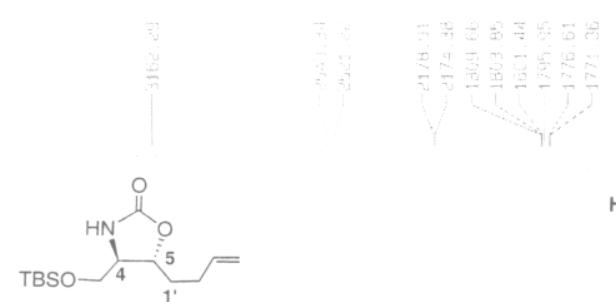

3A (after irradiation of 1' protons decoupling $\mathrm{H}-5$ and $\mathrm{H}-1^{\prime}$ )
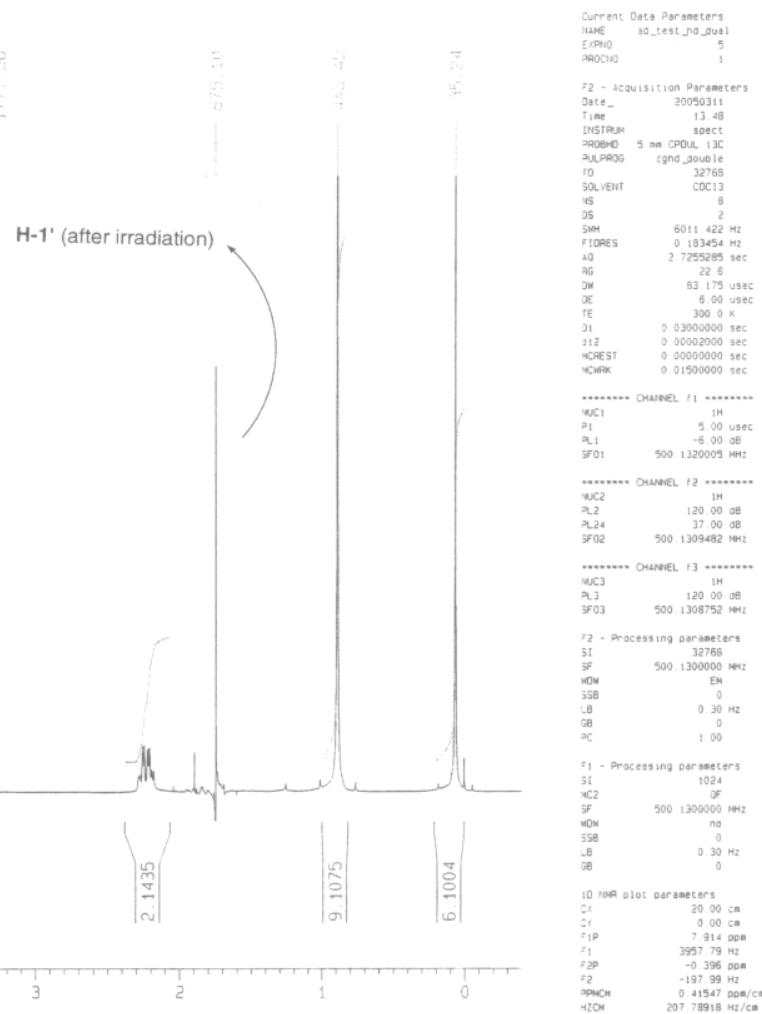

$\mathrm{ppm}$
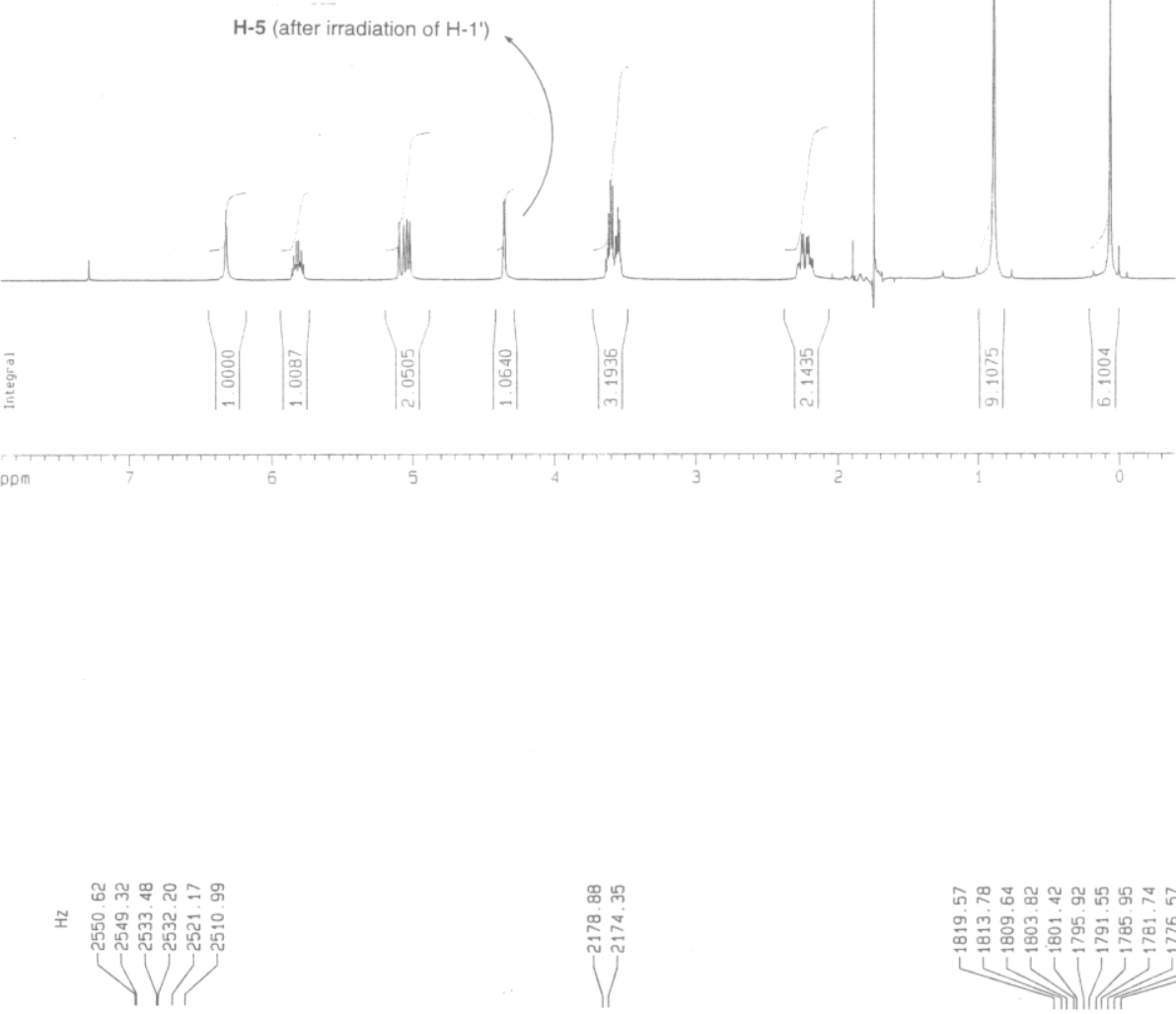

ธั 유 요

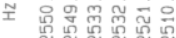

(1)

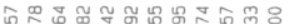

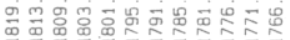
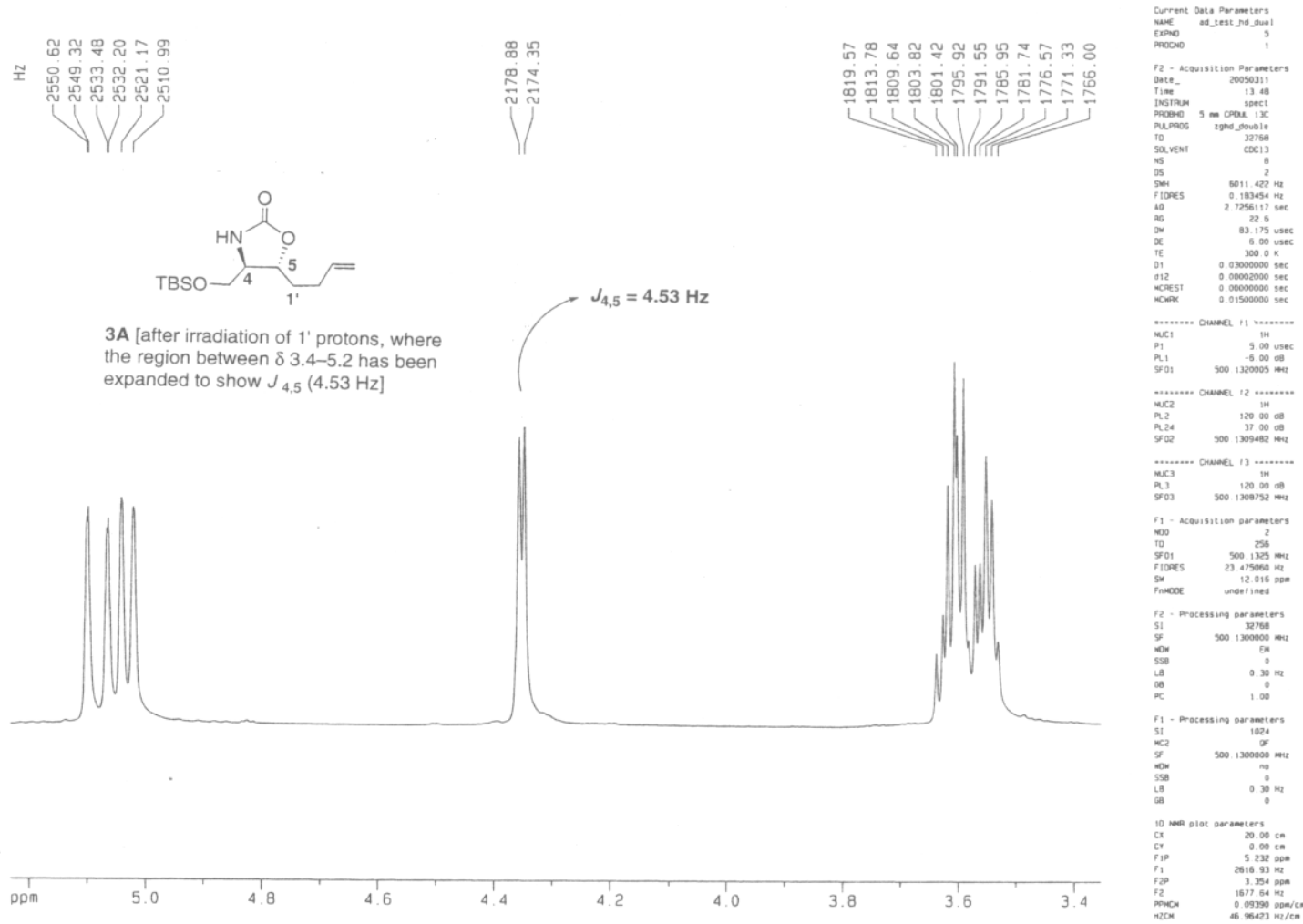

ppm
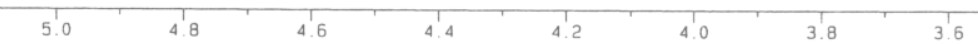
PROTON CDC13
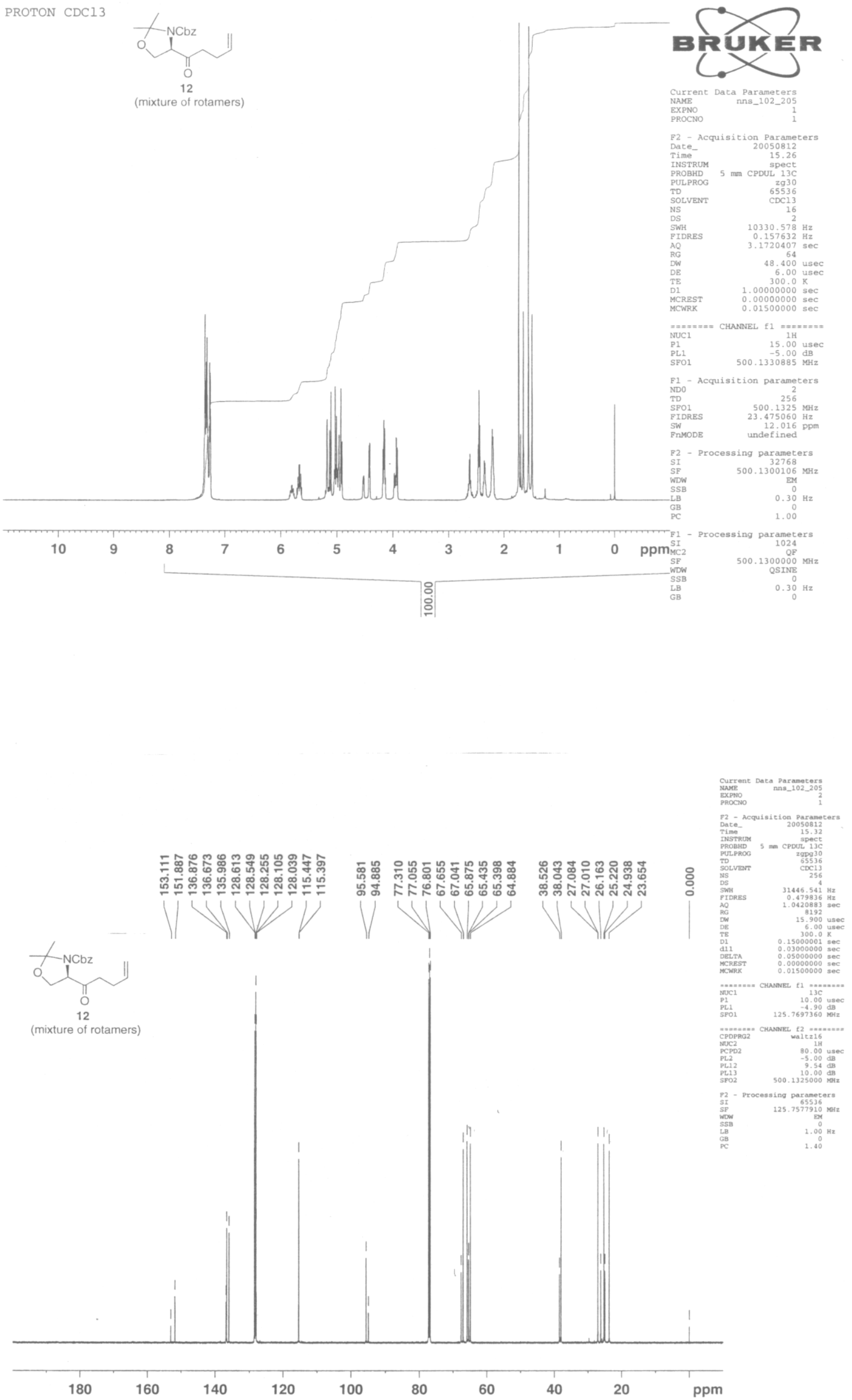

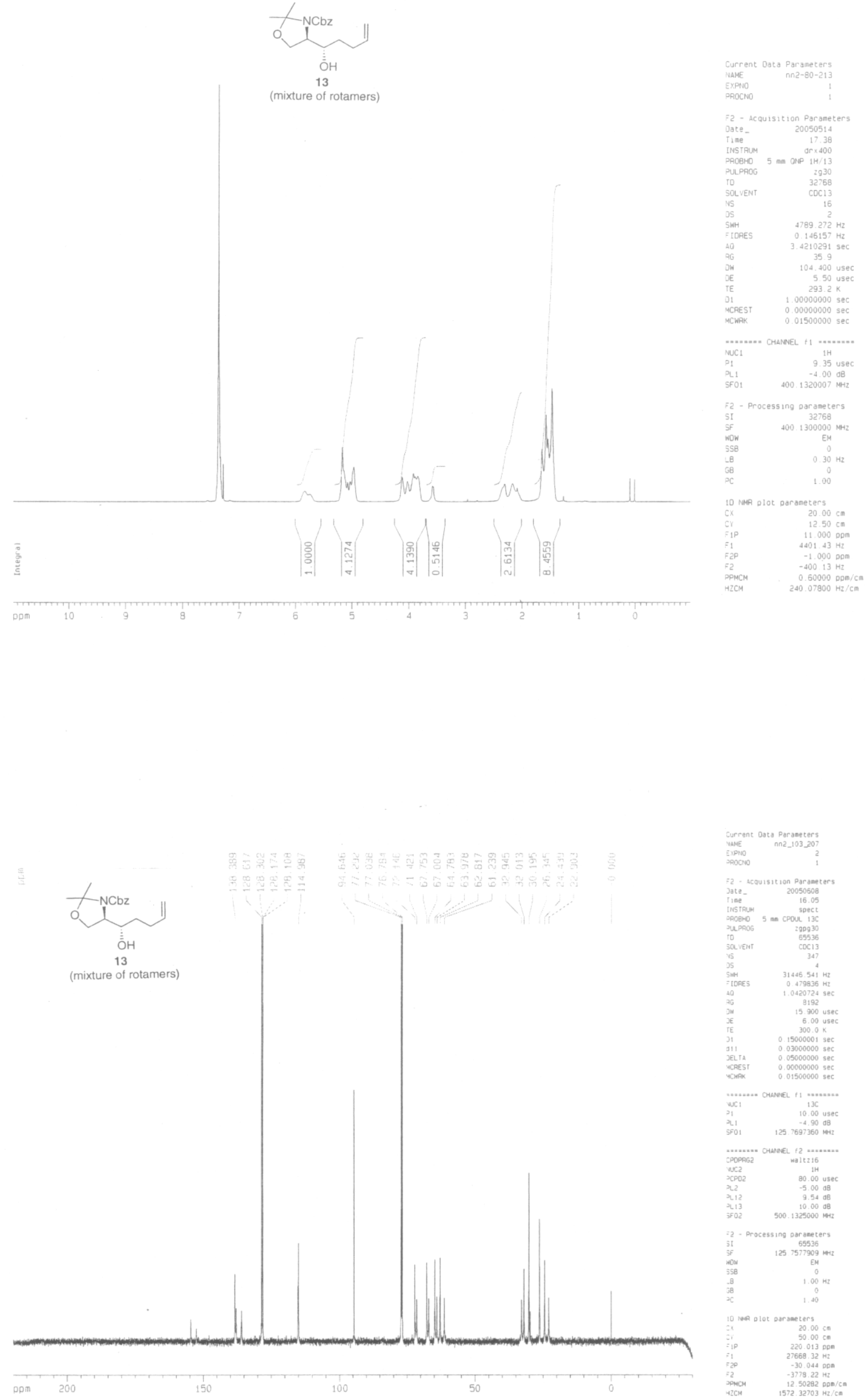


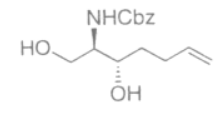

4
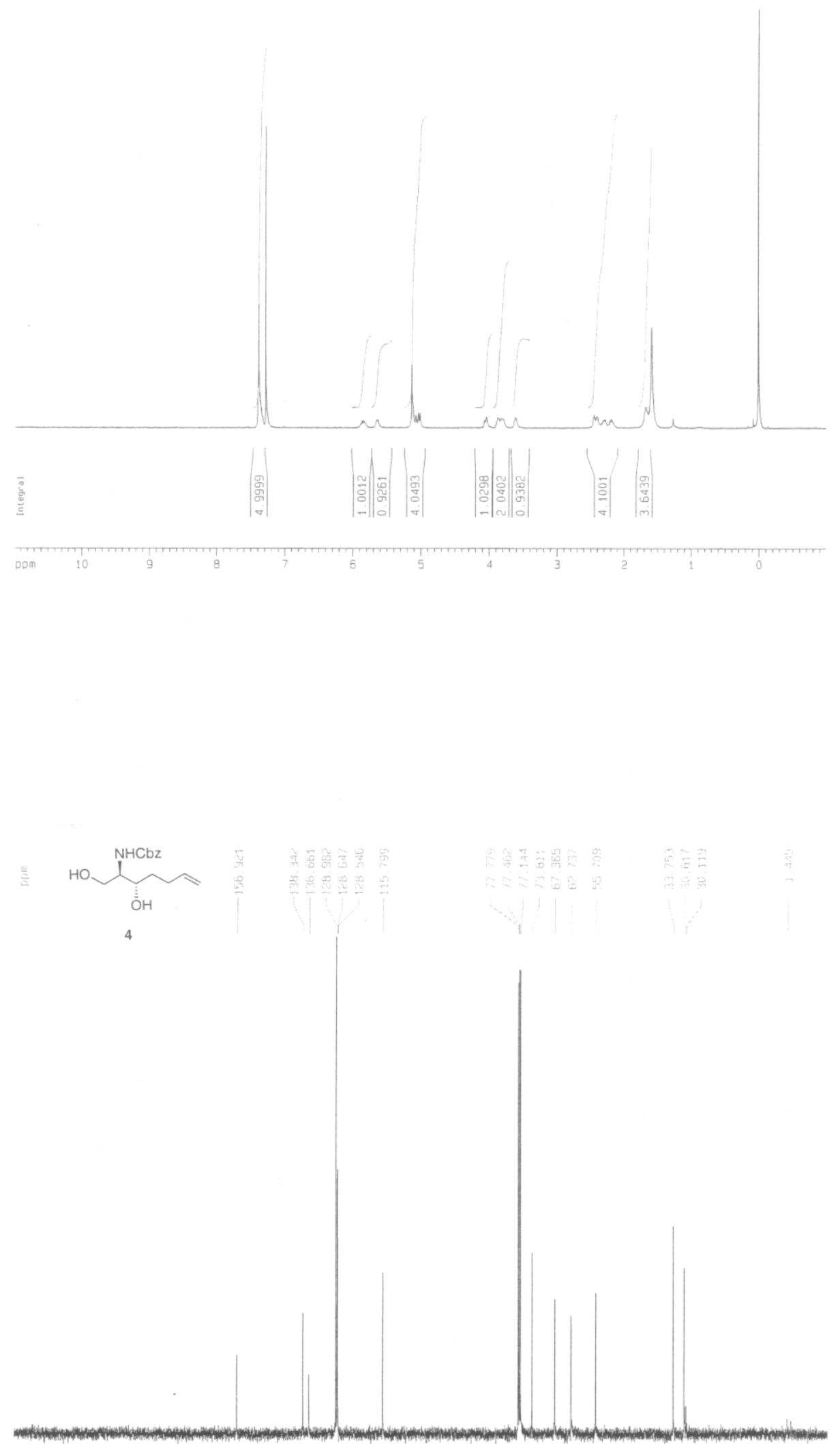

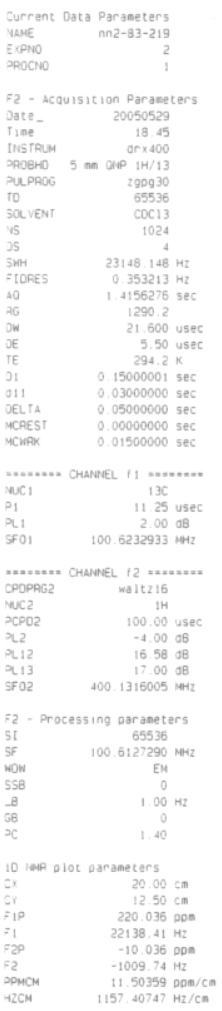




\section{S13}
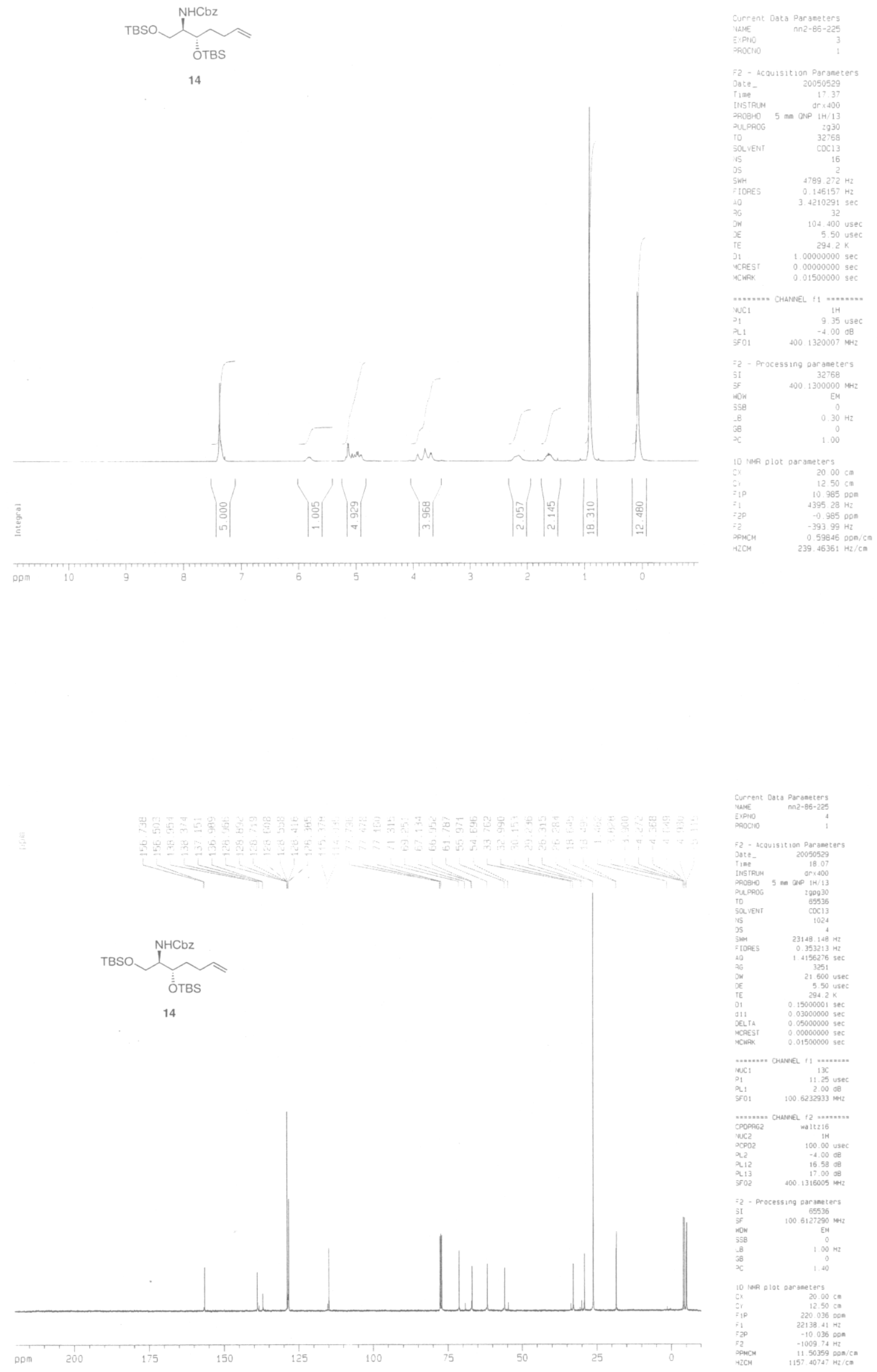

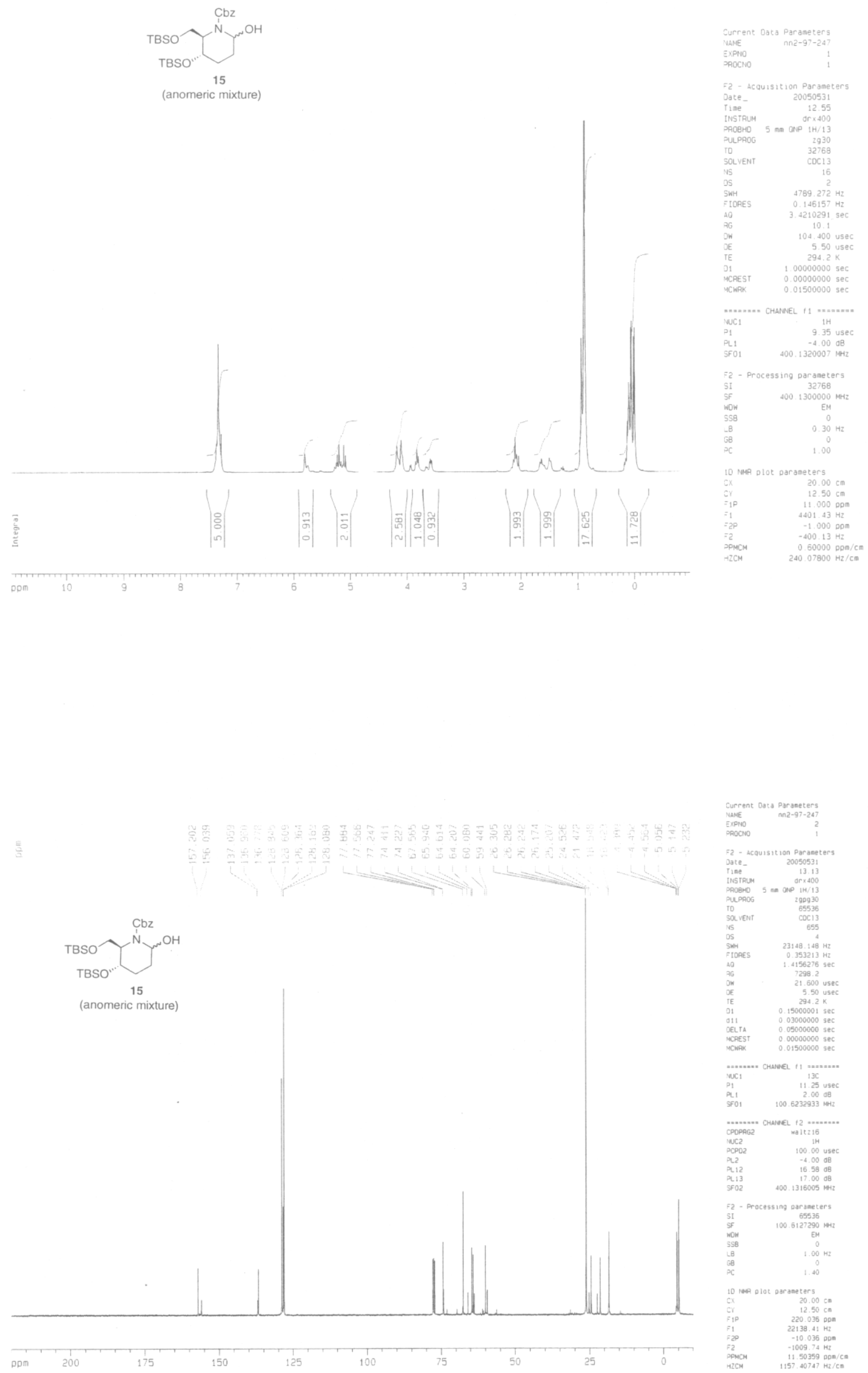

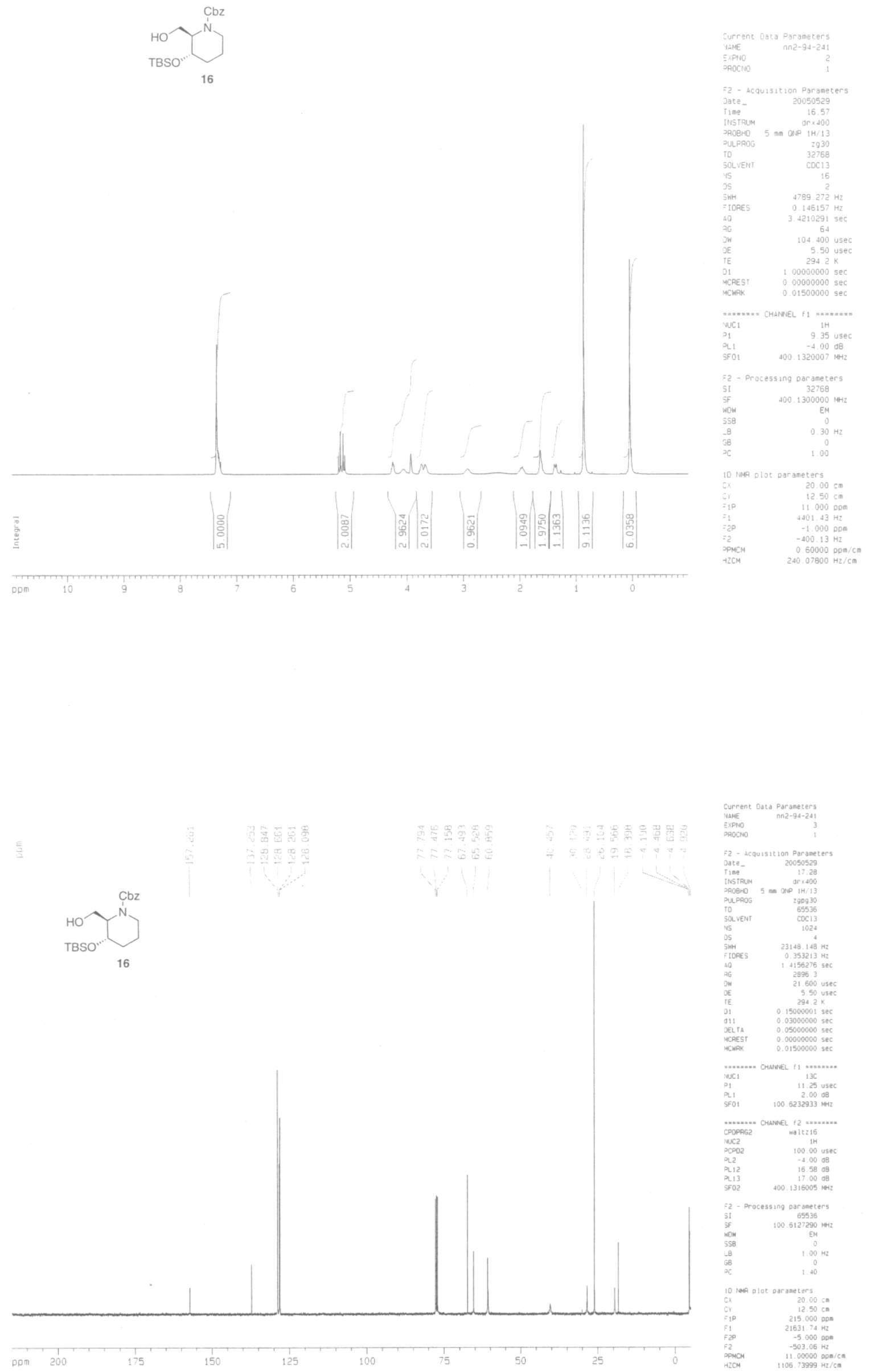

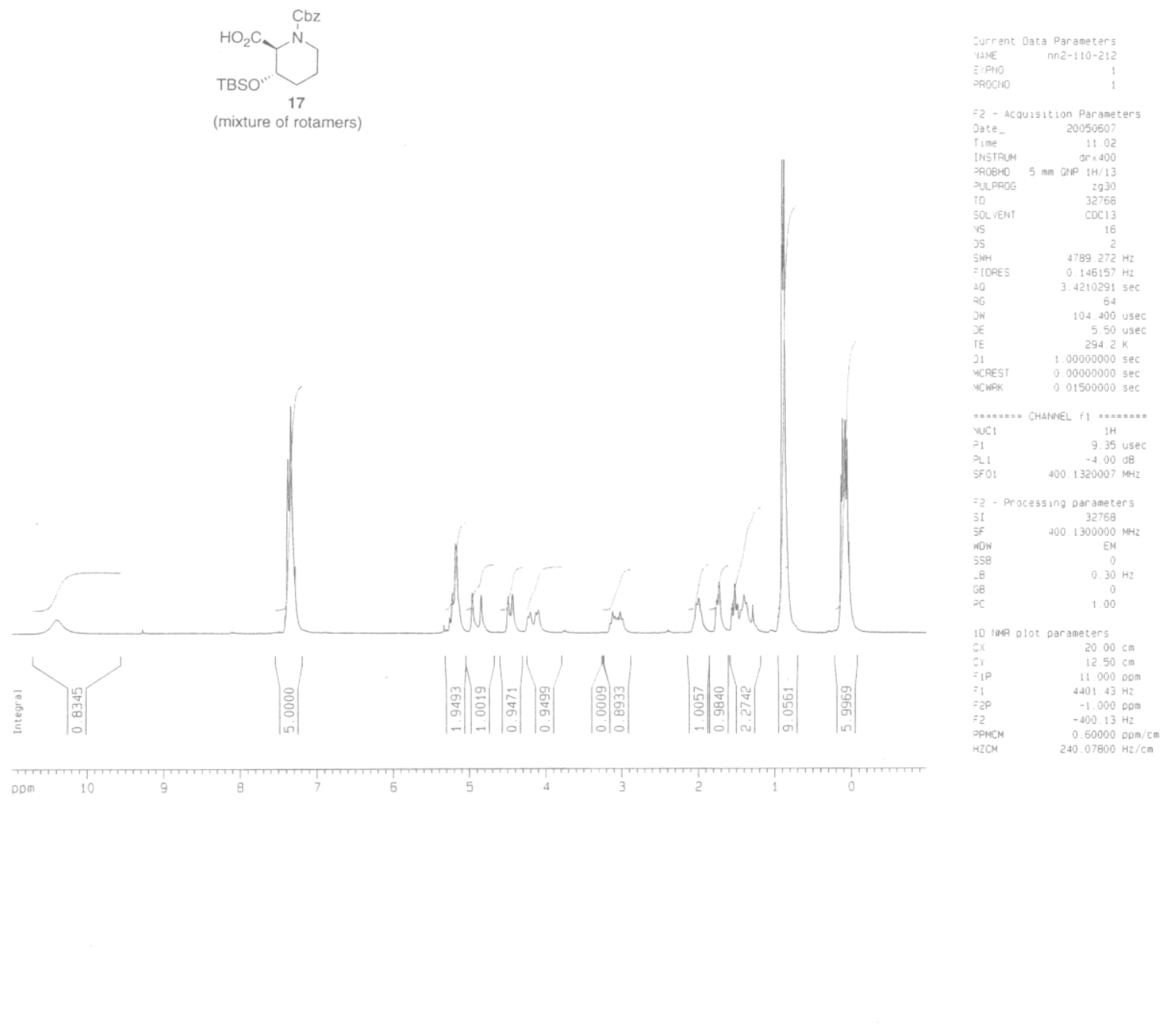

pond
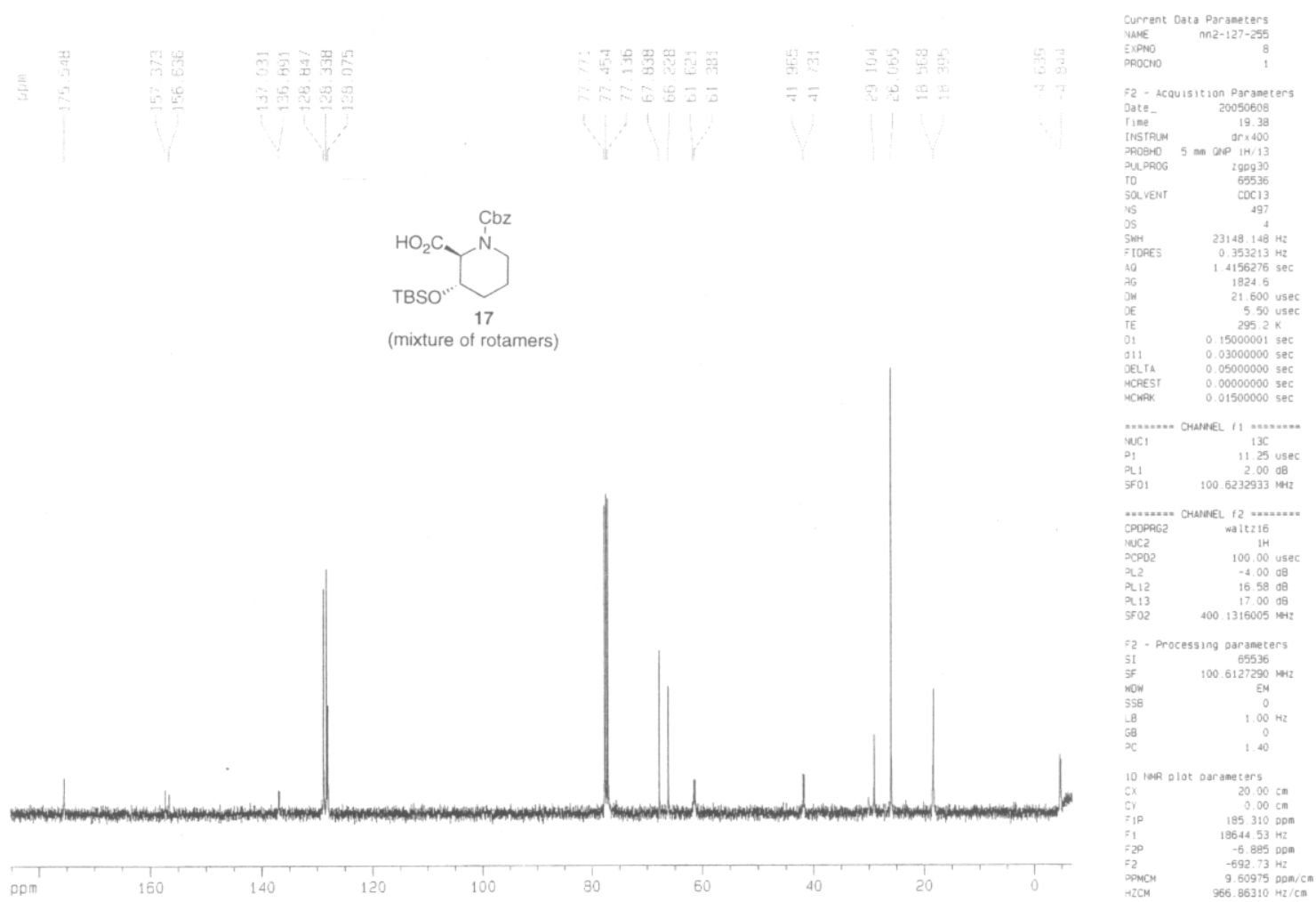

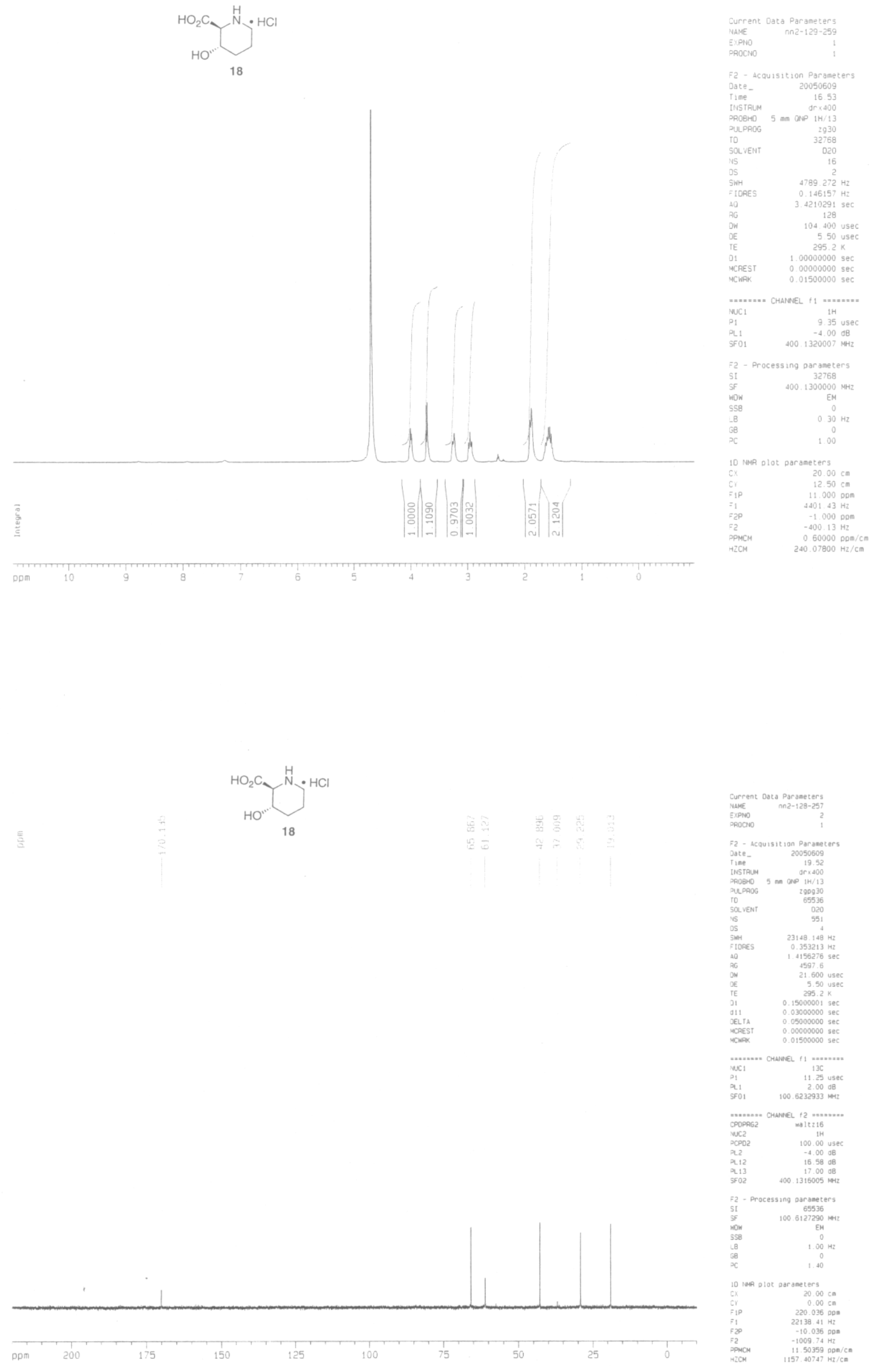

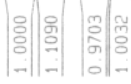

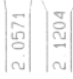

$240.07900 \mathrm{~Hz} / \mathrm{cm}$ 